\title{
Newton method for finding a singularity of a special class of locally Lipschitz continuous vector fields on Riemannian manifolds
}

\author{
Fabiana R. de Oliveira * $\quad$ Orizon P. Ferreira ${ }^{\dagger}$
}

March 19, 2019

\begin{abstract}
In this paper, we extend some results of nonsmooth analysis from Euclidean context to the Riemannian setting. In particular, we discuss the concept and some properties of locally Lipschitz continuous vector fields on Riemannian settings, such as Clarke generalized covariant derivative, upper semicontinuity and Rademacher theorem. We also present a version of Newton method for finding a singularity of a special class of locally Lipschitz continuous vector fields. Under mild conditions, we establish the well-definedness and local convergence of the sequence generated by the method in a neighborhood of a singularity. In particular, a local convergence result for semismooth vector fields is presented. Furthermore, under Kantorovich-type assumptions the convergence of the sequence generated by the Newton method to a solution is established, and its uniqueness in a suitable neighborhood of the starting point is verified.
\end{abstract}

Keywords: Riemannian manifold, locally Lipschitz continuous vector fields, Clarke generalized covariant derivative, semismooth vector field, regularity, Newton method.

AMS Subject Classification: 90C30, 49J52, 90C56.

\section{Introduction}

During the last decade, there has been an increasing the number of papers that have proposed extensions of concepts and techniques of nonsmooth analysis, as well as nonsmooth methods, from Euclidean context to the Riemannian setting, these paper include but not limited to [1, 2, 18, 19, 21, [22, 26]. In these works, some concepts and classical results, such as generalized gradient or Clarke subdifferential, generalized directional derivative, chain rule and Lebourg mean value theorem have been generalized to Riemannian context. In addition, different methods have been proposed for solving nonsmooth optimization problems on Riemannian manifolds. For instance, in [22] was proposed the gradient sampling algorithm for finding a minimizer of nonsmooth locally Lipschitz functions, in [19] was presented a Riemannian trust region method for unconstrained optimization problems, whose objective functions are locally Lipschitz. One the main reason of the increasing interest to develop theoretical and computational tools is the fact that nonsmooth optimization problems arise in a variety of applications, such as in computer vision, robotics, signal processing,

\footnotetext{
*Universidade Federal de Goiás, Goiânia, GO 74001-970, BR (e-mail:fabianardo@gmail.com). The author was supported in part by CAPES.

${ }^{\dagger}$ Universidade Federal de Goiás, Goiânia, GO 74001-970, BR (e-mail:orizon@ufg.br). The author was supported in part by CNPq Grants 305158/2014-7, 408151/2016-1 and FAPEG/GO.
} 
see [2, 3]. Although interest in nonsmooth analysis in the Riemannian setting has increased, there are few papers on nonsmooth vector fields in this context, see [18, 32]. However, as the nonsmooth vector fields can be viewed as a natural generalization of nonsmooth vector-valued maps, we believe that the development of this theory is great interest.

Our purpose in this paper is present a version of Newton method for finding a singularity of locally Lipschitz continuous vector fields. In order to present our method, we first define the Clarke generalized covariant derivative, which can be viewed as a natural generalization to Riemannian setting of Clarke generalized Jacobian, studied in [6]. The concept of Clarke generalized covariant derivative has already appeared in [18, 32. In the present paper, we show its existence using a version of Rademacher theorem in the Riemannian setting, which is one of our contributions.

It is well-known that the Newton method is the method more popular for finding a singularity of a differentiable vector field, the origins of which go back to the work of [34, see also [17, 28, 35, 38, 39]. This method became popular owing to its attractive convergence properties under suitable assumptions. For instance, in all previously cited works the superlinear and/or quadratic local convergence of the sequence generated by the Newton method have been established under invertibility of the covariant derivative of the vector field at its singularity and/or Lipschitz-like conditions on the covariant derivative of the vector field. Recently, in [13] were established local properties of Newton method under invertibility of the covariant derivative of the vector field at its singularity. Basically, in the Newton method the vector field is replaced by an approximation depending on the current iterate, and then the original problem is converted in an approximated problem which can be solved more easily. The solution of this approximated problem is then taken as a new iterate and the process is repeated. The success of Newton method for finding a singularity of a differentiable vector field motivates us to study Newton method for finding a singularity of a locally Lipschitz continuous vector field. The essence of the our method is similar to classical case, however, in the approximated problem we combines the exponential mapping on the manifold with an element of Clarke generalized covariant derivative of the vector field. This is because, the derivative covariant of a locally Lipschitz continuous vector field may not exist. It is worth to pointed out that when the vector field is continuously differentiable our method reduces to the classical Newton method. From the theoretical viewpoint, we present local and semi-local convergence analysis of the proposed method under mild assumptions.

The paper is organized as follows. In Section 2, some notation and basic results are presented. In Section 3, we generalize some results of nonsmooth analysis to Riemannian context, in particular, we establish the Rademacher theorem, introduce the Clarke generalized covariant derivative associated to a locally Lipschitz continuous vector field and study its main properties. In Section 4, we describe the Newton method and establish its convergence theorems. In Section 5, we present a class of examples of locally Lipschitz continuous vector field satisfying the assumptions of the convergence theorems. We conclude the paper with some remarks in Section 6 .

\section{Notation and Auxiliary Results}

In this section, we recall some notations, definitions and basic properties of Riemannian manifolds used throughout the paper. They can be found in many books on Riemannian Geometry, for example, in [25, 33, 37].

A chart on a $n$-dimensional smooth manifold $M$ is a pair $(U, \varphi)$, where $U$ is an open subset of $M$ and the coordinate map $\varphi: U \rightarrow \widehat{U}$ is a smooth homeomorphism from $U$ to an open subset 
$\widehat{U}=\varphi(U) \subseteq \mathbb{R}^{n}$. Let $N$ and $M$ be manifolds of finite dimension and $F: N \rightarrow M$ be a continuous map. We say that $F$ is smooth at $p \in N$, if there exist smooth charts $(U, \varphi)$ containing $p$ and $(W, \psi)$ containing $F(p)$ such that $F(U) \subseteq W$ and the composite mapping $\psi \circ F \circ \varphi^{-1}: \varphi(U) \rightarrow \psi(W)$ is smooth at $\varphi(p)$. The definition of the smoothness of a map $F: N \rightarrow M$ at a point is independent of the choice of charts, see [37, Proposition 6.7]. A diffeomorphism of manifolds is a bijective smooth map $F: N \rightarrow M$ whose inverse $F^{-1}$ is also smooth. According to [37, Proposition 6.10] coordinate maps are diffeomorphisms and, in particular, are continuously differentiable. Let $M$ be a Riemannian manifold with Riemannian metric denoted by $\langle\cdot, \cdot\rangle$ and the corresponding norm by $\|\cdot\|$. The length of a piecewise smooth curve $\gamma:[a, b] \rightarrow M$ joining $p$ to $q$ in $M$, i.e., $\gamma(a)=p$ and $\gamma(b)=q$ is denoted by $\ell(\gamma)$. The Riemannian distance between $p$ and $q$ is defined as $d(p, q)=\inf _{\gamma \in \Gamma_{p, q}} \ell(\gamma)$, where $\Gamma_{p, q}$ is the set of all piecewise smooth curves in $M$ joining points $p$ and $q$. This distance induces the original topology on $M$, namely $(M, d)$ is a complete metric space and the bounded and closed subsets are compact. The open and closed balls of radius $r>0$ centred at $p$ are defined respectively by $B_{r}(p):=\{q \in M: d(p, q)<r\}$ and $B_{r}[p]:=\{q \in M: d(p, q) \leq r\}$. Consider $M$ a $n$-dimensional smooth Riemannian manifold. Denote the tangent space at point $p$ by $T_{p} M$, the tangent bundle by $T M:=\bigcup_{p \in M} T_{p} M$ and a vector field by a mapping $X: M \rightarrow T M$ such that $X(p) \in T_{p} M$. Let $\gamma$ be a curve joining the points $p$ and $q$ in $M$ and let $\nabla$ be the Levi-Civita connection associated to $(M,\langle\cdot, \cdot\rangle)$. For each $t \in[a, b], \nabla$ induces a linear isometry between the tangent spaces $T_{\gamma(a)} M$ and $T_{\gamma(t)} M$, relative to $\langle\cdot, \cdot\rangle$, defined by $P_{\gamma, a, t} v=Y(t)$, where $Y$ is the unique vector field on $\gamma$ such that $\nabla_{\gamma^{\prime}(t)} Y(t)=0$ and $Y(a)=v$. This isometry is called parallel transport along the segment $\gamma$ joining $\gamma(a)$ to $\gamma(t)$. It can be showed that $P_{\gamma, b_{1}, b_{2}} \circ P_{\gamma, a, b_{1}}=P_{\gamma, a, b_{2}}$ and $P_{\gamma, b, a}=P_{\gamma, a, b}^{-1}$. For simplify and convenience, whenever there is no confusion we will consider the notation $P_{\gamma, p, q}$ instead of $P_{\gamma, a, b}$, where $\gamma$ is a segment joining $p$ to $q$ with $\gamma(a)=p$ and $\gamma(b)=q$. We will use the short notation $P_{p q}$ instead of $P_{\gamma, p, q}$ whenever there exists an unique geodesic segment joining $p$ to $q$. For any $n$-dimensional smooth manifold $M$; the tangent bundle $T M$ has a natural topology and smooth structure that make it into a $2 n$-dimensional smooth manifold. With respect to this structure, the projection $\pi: T M \rightarrow M$ is smooth, see [27, Proposition 3.18]. The standard Riemannian distance $d_{T M}$ on the tangent bundle $T M$ can be defined as follows: given $u, v \in T M$, then $d_{T M}$ is defined by

$$
d_{T M}(u, v):=\inf \left\{\sqrt{\ell^{2}(\gamma)+\left\|P_{\gamma, \pi u, \pi v} u-v\right\|^{2}}: \gamma \in \Gamma_{\pi u, \pi v}\right\},
$$

where $\Gamma_{\pi u, \pi v}$ is the set of all piecewise smooth curves in $M$ joining the points $\pi u$ to $\pi v$, whose derivative is never zero, see [5, Appendix, p. 240]. A vector field $Y$ along a smooth curve $\gamma$ in $M$ is said to be parallel when $\nabla_{\gamma^{\prime}} Y=0$. If $\gamma^{\prime}$ itself is parallel, we say that $\gamma$ is a geodesic. The geodesic equation $\nabla_{\gamma^{\prime}} \gamma^{\prime}=0$ is a second-order nonlinear ordinary differential equation, so the geodesic $\gamma$ is determined by its position $p$ and velocity $v$ at $p$. It is easy to check that $\left\|\gamma^{\prime}\right\|$ is constant. The restriction of a geodesic to a closed bounded interval is called a geodesic segment. A geodesic segment joining $p$ to $q$ in $M$ is said to be minimal if its length is equal to $d(p, q)$ and, in this case, it will be denoted by $\gamma_{p q}$. A Riemannian manifold is complete if its geodesics $\gamma(t)$ are defined for any value of $t \in \mathbb{R}$. The Hopf-Rinow theorem asserts that any pair of points in a complete Riemannian manifold $M$ can be joined by a (not necessarily unique) minimal geodesic segment. From now on, $M$ denotes a n-dimensional smooth and complete Riemannian manifold. Due to the completeness of the Riemannian manifold $M$, the exponential map at $p, \exp _{p}: T_{p} M \rightarrow M$ can be given by $\exp _{p} v=\gamma(1)$, where $\gamma$ is the geodesic defined by its position $p$ and velocity $v$ at $p$ and $\gamma(t)=\exp _{p}(t v)$ for any value of $t$. The inverse of the exponential map (if exists) is denote by $\exp _{p}^{-1}$. Let $p \in M$, the injectivity radius of $M$ at $p$ is defined by $r_{p}:=\sup \left\{r>0: \exp _{p_{B_{r}\left(0_{p}\right)}}\right.$ is a diffeomorphism $\}$, where $0_{p}$ denotes the origin of $T_{p} M$ and $B_{r}\left(0_{p}\right):=\left\{v \in T_{p} M:\left\|v-0_{p}\right\|<r\right\}$. A neighborhood $\mathcal{W}$ of 
$p \in M$ is said to be normal neighborhood of $p$ if there exists a neighborhood $\mathcal{U}$ of the origin in $T_{p} M$ such that $\exp _{p}: \mathcal{U} \rightarrow \mathcal{W}$ is a diffeomorphism. Furthermore, if $\mathcal{W}$ is a normal neighborhood of each of its points, then $\mathcal{W}$ is said to be totally normal neighborhood.

Remark 1. For $\bar{p} \in M$, the above definition implies that if $0<\delta<r_{\bar{p}}$, then $\exp _{\bar{p}} B_{\delta}\left(0_{\bar{p}}\right)=B_{\delta}(\bar{p})$ is a totally normal neighborhood. Hence, for all $p, q \in B_{\delta}(\bar{p})$, there exists a unique geodesic segment $\gamma$ joining $p$ to $q$, which is given by $\gamma_{p q}(t)=\exp _{p}\left(t \exp _{p}^{-1} q\right)$, for all $t \in[0,1]$ and $d(p, q)=\left\|\exp _{p}^{-1} q\right\|$.

Next we present a quantity, which plays an important role in the sequel, it was defined in [8].

Definition 2. Let $p \in M$ and $r_{p}$ be the radius of injectivity of $M$ at $p$. Define the quantity

$$
K_{p}:=\sup \left\{\frac{d\left(\exp _{q} u, \exp _{q} v\right)}{\|u-v\|}: q \in B_{r_{p}}(p), u, v \in T_{q} M, u \neq v,\|v\| \leq r_{p},\|u-v\| \leq r_{p}\right\} .
$$

In the next remark, we examine that under suitable conditions is possible to estimate the value of $K_{p}$.

Remark 3. This number $K_{p}$ measures how fast the geodesics spread apart in $M$. In particular, when $u=0$ or more generally when $u$ and $v$ are on the same line through $0, d\left(\exp _{q} u, \exp _{q} v\right)=$ $\|u-v\|$. Hence, $K_{p} \geq 1$, for all $p \in M$. When $M$ has non-negative sectional curvature, the geodesics spread apart less than the rays [9, Chapter 5], i.e., $d\left(\exp _{p} u, \exp _{p} v\right) \leq\|u-v\|$ and, in this case, $K_{p}=1$ for all $p \in M$.

The directional derivative of $X$ at $p$ in the direction $v \in T_{p} M$ is defined by

$$
\nabla X(p, v)=\lim _{t \rightarrow 0^{+}} \frac{1}{t}\left[P_{\exp _{p}(t v) p} X\left(\exp _{p}(t v)\right)-X(p)\right] \in T_{p} M,
$$

whenever the limit exists, where $P_{\exp _{p}(t v) p}$ denotes the parallel transport along $\gamma(t)=\exp _{p}(t v)$. If this directional derivative exists for every $v$, then $X$ is said to be directionally differentiable at $p$. Denote by $\mathcal{X}(M)$ the space of the differentiable vector fields on $M$. For each $X \in \mathcal{X}(M)$, the covariant derivative of $X$ determined by the Levi-Civita connection $\nabla$ defines at each $p \in M$ a linear mapping $\nabla X(p): T_{p} M \rightarrow T_{p} M$ given by $\nabla X(p) v:=\nabla_{Y} X(p)$, where $Y$ is a vector field such that $Y(p)=v$. Furthermore, $\nabla X(p, v)=\nabla X(p) v$, see [36, Proposition 3, p. 234]. To state the next result we need to define the norm of a linear mapping.

Definition 4. Let $p \in M$, the norm of a linear mapping $A: T_{p} M \rightarrow T_{p} M$ is defined by

$$
\|A\|:=\sup \left\{\|A v\|: v \in T_{p} M,\|v\|=1\right\} .
$$

We end this section with the well-known Banach Lemma. For a prove of it see [30, Lemma 2.3.2].

Lemma 5. Let $A, B$ be a linear operators in $T_{p} M$. If $A$ is non-singular and $\left\|A^{-1}\right\|\|B-A\|<1$, then $B$ is non-singular and

$$
\left\|B^{-1}\right\| \leq \frac{\left\|A^{-1}\right\|}{1-\left\|A^{-1}(B-A)\right\|} .
$$




\section{Nonsmooth Analysis}

The goal of this section is extend some basic results of nonsmooth analysis from linear context to Riemannian setting. In particular, we study the basic properties of the locally Lipschitz continuous vector fields in Riemannian setting, a generalization of Rademacher theorem and introduce the concept of Clarke generalized covariant derivative to this new context. A comprehensive study of nonsmooth analysis in linear context can be found in [6]. We begin with the definition of locally Lipschitz continuous vector fields, this concept was introduced in [7] for gradient vector fields and its extension to general vector fields can be found in [5, p. 241].

Definition 6. A vector field $X$ on $M$ is said to be Lipschitz continuous on $\Omega \subset M$, if there exists a constant $L>0$ such that for $p, q \in M$ and all $\gamma$ geodesic segment joining $p$ to $q$, there holds

$$
\left\|P_{\gamma, p, q} X(p)-X(q)\right\| \leq L \ell(\gamma), \quad \forall p, q \in \Omega .
$$

Given $p \in M$, if there exists $\delta>0$ such that $X$ is Lipschitz continuous on $B_{\delta}(p)$, then $X$ is said to be Lipschitz continuous at $p$. Moreover, if for all $p \in M, X$ is Lipschitz continuous at $p$, then $X$ is said to be locally Lipschitz continuous on $M$.

Let $d_{T M}$ be the Riemannian distance on $T M$. Let us define the concept of Lipschitz continuity of vector field as a map between the metric spaces $(M, d)$ and $\left(T M, d_{T M}\right)$. The formal definition is:

Definition 7. A vector field $X$ on $M$ is said to be metrically Lipschitz continuous on $\Omega \subset M$, if there exists a constant $L>0$ such that

$$
d_{T M}(X(p), X(q)) \leq L d(p, q), \quad \forall p, q \in \Omega .
$$

Given $p \in M$, if there exists $\delta>0$ such that $X$ is metrically Lipschitz continuous on $B_{\delta}(p)$, then $X$ is said to be metrically Lipschitz continuous at $p$. Moreover, if for all $p \in M, X$ is metrically Lipschitz continuous at $p$, then $X$ is said to be locally metric Lipschitz continuous on $M$.

It is immediate from the last definition that all metrically Lipschitz continuous vector fields are continuous. In the next result, we present a relationship between two above definitions.

Theorem 8. If $X$ is Lipschitz continuous with constant $L>0$, then $X$ is also metrically Lipschitz continuous with constant $\sqrt{1+L^{2}}$. As a consequence, if $X$ is locally Lipschitz continuous on $M$, then $X$ is also locally metric Lipschitz continuous on $M$.

Proof. Since $M$ is a complete manifold, $\pi X(p)=p$ and $\pi X(q)=q$, it follows from (1) that

$$
d_{T M}(X(p), X(q)) \leq \sqrt{d^{2}(p, q)+\left\|P_{\gamma, p, q} X(p)-X(q)\right\|^{2}}, \quad \forall p, q \in M,
$$

where $\gamma$ is the minimal geodesic segment joining $p$ to $q$. Considering that $X$ is Lipschitz continuous with constant $L>0$ from Definition 6 we have $\left\|P_{\gamma, p, q} X(p)-X(q)\right\| \leq L d(p, q)$ for all $p, q \in M$. Hence, inequality (3) becomes

$$
d_{T M}(X(p), X(q)) \leq \sqrt{1+L^{2}} d(p, q),
$$

for all $p, q \in M$. Consequently, by using Definition 7 we conclude that $X$ is metrically Lipschitz continuous with constant $\sqrt{1+L^{2}}$. Therefore, the proof of the first part is complete. The proof of the second part is similar. 
In the next definition, we present the notion of sets of measure zero to manifolds [27, 33].

Definition 9. A subset $E \subseteq M$ has measure zero in $M$ if for every smooth chart $(U, \varphi)$ for $M$, the subset $\varphi(E \cap U) \subseteq \mathbb{R}^{n}$ has $n$-dimensional measure zero.

Let $X$ be a locally Lipschitz continuous vector field on $M$. Throughout of the paper, $\mathcal{D}_{X}$ is the set defined by $\mathcal{D}_{X}:=\{p \in M: X$ is differentiable at $p\}$. Locally Lipschitz continuous vector fields are in general non-differentiable, however, they are almost everywhere differentiable with respect to the Riemannian measure (see the concept of Riemannian measure in [33, p. 61]), i.e., the set $M \backslash \mathcal{D}_{X}$ has measure zero. This result follows from Rademacher theorem. A version of this theorem for locally Lipschitz continuous vector fields is given below.

Theorem 10. If $X$ is a locally Lipschitz continuous vector field on $M$, then $X$ is almost everywhere differentiable on $M$.

Proof. Since $M$ is a $n$-dimensional smooth manifold then $T M$ is $2 n$-dimensional smooth manifold. First note that Theorem 8 implies that $X$ is continuous. Let $(U, \varphi)$ and $(W, \psi)$ be smooth charts for $M$ and $T M$, respectively, such that $X(U) \subseteq W$ and consider the composite mapping $\psi \circ X \circ$ $\varphi^{-1}: \varphi(U) \rightarrow \psi(W)$. We proceed to prove that the mapping $\psi \circ X \circ \varphi^{-1}$ is locally Lipschitz continuous on $\varphi(U)$. According to [37, Proposition 6.10] we have that the coordinate mappings $\varphi^{-1}: \varphi(U) \rightarrow U$ and $\psi: W \rightarrow \psi(W)$ are diffeomorphisms and, in particular, continuously differentiable. Take $z \in \varphi(U)$ and $\rho>0$ such that $B_{\rho}[z] \subset \varphi(U)$. Since $B_{\rho}[z]$ is compact and the derivative of $\varphi^{-1}$ is continuous in $B_{\rho}[z]$, from Mean Value Inequality, see [1, Theorem 2.14], there exists $L_{1}>0$ such that $d\left(\varphi^{-1}(x), \varphi^{-1}(y)\right) \leq L_{1} \hat{d}(x, y)$, for all $x, y \in B_{\rho}[z]$, where $\hat{d}$ is the Euclidean distance in $\mathbb{R}^{n}$. On the other hand, Theorem 8 implies that $X$ is locally metric Lipschitz continuous on $\varphi(U)$, then shrinking $\rho>0$ if necessary, we conclude that there exists $L_{2}>0$ such that $d_{T M}\left(X \circ \varphi^{-1}(x), X \circ \varphi^{-1}(y)\right) \leq L_{2} d\left(\varphi^{-1}(x), \varphi^{-1}(y)\right)$, for all $x, y \in B_{\rho}[z]$. Since $X\left(\varphi^{-1}\left(B_{\rho}[z]\right)\right)$ is compact and the derivative of $\psi$ is continuous in $X\left(\varphi^{-1}\left(B_{\rho}[z]\right)\right)$ again using Mean Value Inequality, see [1, Theorem 2.14], there exists $L_{3}>0$ such that

$$
\tilde{d}\left(\psi \circ X \circ \varphi^{-1}(x), \psi \circ X \circ \varphi^{-1}(y)\right) \leq L_{3} d_{T M}\left(X \circ \varphi^{-1}(x), X \circ \varphi^{-1}(y)\right), \quad \forall x, y \in B_{\rho}[z],
$$

where $\tilde{d}$ is the Euclidean distance in $\mathbb{R}^{2 n}$. Combining the three last inequalities we obtain that

$$
\tilde{d}\left(\psi \circ X \circ \varphi^{-1}(x), \psi \circ X \circ \varphi^{-1}(y)\right) \leq \widetilde{L} \hat{d}(x, y), \quad \forall x, y \in B_{\rho}[z],
$$

where $\widetilde{L}=L_{1} L_{2} L_{3}>0$. Hence, $\psi \circ X \circ \varphi^{-1}$ is locally Lipschitz continuous on $\varphi(U) \subseteq \mathbb{R}^{n}$. Therefore, from Rademacher theorem, see [11, Theorem 2, p. 81], we have that $\psi \circ X \circ \varphi^{-1}$ is almost everywhere differentiable on $\varphi(U)$. Since the charts $(U, \varphi)$ and $(W, \psi)$ are arbitrary, we conclude that $X$ is almost everywhere differentiable on $M$.

Now, we introduce the Clarke generalized covariant derivative of a locally Lipschitz continuous vector field and explore some of its properties. For a comprehensive study about Clarke generalized Jacobian in linear space, see [6].

Definition 11. The Clarke generalized covariant derivative of a locally Lipschitz continuous vector field $X$ is a set-valued mapping $\partial X: M \rightrightarrows T M$ defined as

$$
\partial X(p):=\operatorname{co}\left\{H \in \mathcal{L}\left(T_{p} M\right): \exists\left\{p_{k}\right\} \subset \mathcal{D}_{X}, \lim _{k \rightarrow+\infty} p_{k}=p, H=\lim _{k \rightarrow+\infty} P_{p_{k} p} \nabla X\left(p_{k}\right)\right\},
$$

where "co" represents the convex hull and $\mathcal{L}\left(T_{p} M\right)$ denotes the vector space consisting of all linear operator from $T_{p} M$ to $T_{p} M$. 
From Definition [11 and [13, Corollary 3.1], it is clear that if $X$ is differentiable near $p$, and its covariant derivative is continuous at $p$, then $\partial X(p)=\{\nabla X(p)\}$. Otherwise, $\partial X(p)$ could contain other elements different from $\nabla X(p)$, even if $X$ is differentiable at $p$, see [6, Example 2.2.3]. In the following proposition, we shall show important results of the Clarke generalized covariant derivative. In particular, that the set $\partial X(p)$ is nonempty for all $p \in M$, and that $\partial X$ is locally bounded and closed, which is a generalization of [6. Proposition 2.6.2, items (a), (b) and (c)]. These results will be very useful throughout this paper. Similar results have already been extended to functions defined in $M$, see [20, Theorem 2.9].

Proposition 12. Let $X$ be locally Lipschitz continuous vector field on $M$. The following statements are valid for any $p \in M$ :

(i) $\partial X(p)$ is a nonempty, convex and compact subset of $\mathcal{L}\left(T_{p} M\right)$;

(ii) the set-valued mapping $\partial X: M \rightrightarrows T M$ is locally bounded, i.e., for all $\delta>0$, there exists a $L>0$ such that for all $q \in B_{\delta}(p)$ and $V \in \partial X(q)$, there holds $\|V\| \leq L$;

(iii) the mapping $\partial X$ is upper semicontinuous at p, i.e., for every scalar $\epsilon>0$, there exists a $0<\delta<r_{p}$, and such that for all $q \in B_{\delta}(p)$,

$$
P_{q p} \partial X(q) \subset \partial X(p)+B_{\epsilon}(0),
$$

where $B_{\epsilon}(0):=\left\{v \in T_{p} M:\|v\|<\epsilon\right\}$. Consequently, the set-valued mapping $\partial X$ is closed at $p$, i.e., if $\lim _{k \rightarrow+\infty} p_{k}=p, V_{k} \in \partial X\left(p_{k}\right)$, for all $k=0,1, \ldots$, and $\lim _{k \rightarrow+\infty} P_{p_{k} p} V_{k}=V$, then $V \in \partial X(p)$.

Proof. To prove item $(i)$ we define the auxiliary set

$$
\partial_{B} X(p):=\left\{H \in \mathcal{L}\left(T_{p} M\right): \exists\left\{p_{k}\right\} \subset \mathcal{D}_{X}, \lim _{k \rightarrow+\infty} p_{k}=p, H=\lim _{k \rightarrow+\infty} P_{p_{k} p} \nabla X\left(p_{k}\right)\right\} .
$$

Owing to the fact that $T_{p} M$ is finite dimensional and $\partial X(p)$ is the convex hull in $\mathcal{L}\left(T_{p} M\right)$ of the set $\partial_{B} X(p)$, thus $\partial X(p)$ must be convex. Our next goal is to prove that $\partial X(p)$ is compact. Due to the convex hull of a compact set be compact, it is sufficient to prove that $\partial_{B} X(p)$ is bounded and closed. Our first task is to prove that $\partial_{B} X(p)$ is bounded. For this, take $p \in \mathcal{D}_{X}$ and $v \in T_{p} M$. Since $\nabla X(p) v=\nabla X(p, v)$ using (2), the fact that $X$ is locally Lipschitz continuous on $M$ and the definition of exponential mapping we obtain that

$$
\|\nabla X(p) v\|=\lim _{t \rightarrow 0^{+}}\left\|\frac{1}{t}\left[P_{\exp _{p}(t v) p} X\left(\exp _{p}(t v)\right)-X(p)\right]\right\| \leq L\|v\|,
$$

where $L>0$ is the Lipschitz constant of $X$ around $p$. Hence, from Definition 4 we conclude that $\|\nabla X(p)\| \leq L$, which implies that $\partial_{B} X(p)$ is bounded. To prove that $\partial_{B} X(p)$ is closed, let $\left\{H_{\ell}\right\}$ be a sequence in $\partial_{B} X(p)$ such that $\lim _{\ell \rightarrow+\infty} H_{\ell}=H$. Since $\left\{H_{\ell}\right\} \subset \partial_{B} X(p)$, there is a sequence $\left\{p_{k, \ell}\right\}$ such that $\lim _{k \rightarrow+\infty} p_{k, \ell}=p$, and $\lim _{k \rightarrow+\infty} P_{p_{k, \ell}} \nabla X\left(p_{k, \ell}\right)=H_{\ell}$, for each fixed $\ell$. Therefore, $\lim _{k \rightarrow+\infty} p_{k, k}=p$ and $\lim _{k \rightarrow+\infty} P_{p_{k, k} p} \nabla X\left(p_{k, k}\right)=H$, and then $H \in \partial_{B} X(p)$. Consequently $\partial_{B} X(p)$ is compact set. To prove that $\partial X(p)$ is a nonempty set, we first note that Theorem 10 implies that $X$ is almost everywhere differentiable on $M$, i.e., the set $M \backslash \mathcal{D}_{X}$ has measure zero. According to [27, Proposition 6.8], $\mathcal{D}_{X}$ is dense in $M$. Then, for any fixed point $p \in M$ there exists $\left\{p_{k}\right\} \subset \mathcal{D}_{X}$ that converges to $p$. Since the covariant derivative of $X$ at points differentiable near $p$ are bounded in norm by the Lipschitz constant and the parallel transport is an isometry we 
conclude that $\left\{P_{p_{k} p} \nabla X\left(p_{k}\right)\right\}$ must have at least one accumulation point and thus $\partial X(p)$ is indeed a nonempty set. To prove item (ii), take $\delta>0, p \in M$ and $L>0$ the Lipschitz constant of $X$ around $p$. The same argument used to prove item $(i)$ shows that $\|\nabla X(\bar{q})\| \leq L$ for all $\bar{q} \in B_{\delta}(p) \cap \mathcal{D}_{X}$. Let $q \in B_{\delta}(p)$ and $V \in \partial X(q)$. Then, there exist $H_{1}, \ldots, H_{m} \in \partial_{B} X(q)$ and $\alpha_{1}, \ldots, \alpha_{m} \in[0,1]$ such that $V=\sum_{\ell=1}^{m} \alpha_{\ell} H_{\ell}$ and $\sum_{\ell=1}^{m} \alpha_{\ell}=1$. Since $H_{1}, \ldots, H_{m} \in \partial_{B} X(q)$ then, there exists $\left\{q_{k, \ell}\right\} \subset B_{\delta}(p) \cap \mathcal{D}_{X}$ with $\lim _{k \rightarrow+\infty} q_{k, \ell}=q$ such that $V=\sum_{\ell=1}^{m} \alpha_{\ell} \lim _{k \rightarrow+\infty} P_{q_{k, \ell} q} \nabla X\left(q_{k, \ell}\right)$. Owing to $\left\{q_{k, \ell}\right\} \subset B_{\delta}(p) \cap \mathcal{D}_{X}$ we have $\left\|\nabla X\left(q_{k, \ell}\right)\right\| \leq L$. Therefore, using that the parallel transport is an isometry we conclude that

$$
\|V\|=\left\|\sum_{\ell=1}^{m} \alpha_{\ell} \lim _{k \rightarrow+\infty} P_{q_{k, \ell} q} \nabla X\left(q_{k, \ell}\right)\right\| \leq \sum_{\ell=1}^{m} \alpha_{\ell} \lim _{k \rightarrow+\infty}\left\|P_{q_{k, \ell} q} \nabla X\left(q_{k, \ell}\right)\right\| \leq L,
$$

which is the desired inequality. To prove item (iii), suppose by contradiction that, for a given $\epsilon>0$ and all $0<\delta<r_{p}$ there exists $q \in B_{\delta}(p)$ such that $P_{q p} \partial X(q) \not \subset \partial X(p)+B_{\epsilon}(0)$. Then, there exists a sequence $\left\{q_{k}\right\} \subset \mathcal{D}_{X}$ such that $\lim _{k \rightarrow+\infty} q_{k}=p$ and $P_{q_{k} p} \nabla X\left(q_{k}\right) \notin \partial X(p)+B_{\epsilon}(0)$. On the other hand, the item $(i i)$ implies that $\partial X$ is locally bounded. Since the parallel transport is an isometry we have $\left\{P_{q_{k} p} \nabla X\left(q_{k}\right)\right\}$ is bounded. Thus, we can extract $\left\{P_{q_{k_{\ell}} p} \nabla X\left(q_{k_{\ell}}\right)\right\}$ a convergent subsequence of $\left\{P_{q_{k} p} \nabla X\left(q_{k}\right)\right\}$, let us say that $\left\{P_{q_{k_{\ell}} p} \nabla X\left(q_{k_{\ell}}\right)\right\}$ converges to some $H$. From Definition 11 we obtain that $H \in \partial X(p)$, which is a contradiction. Therefore, $\partial X$ is upper semicontinuous at $p$. The last part of the item $(\mathrm{iii})$ is an immediate consequence of the first part and the proof is complete.

\section{The Newton Method}

In this section, we present the Newton method for finding a singularity of a vector field $X$ on $M$, i.e., to solve the following problem

$$
\text { find } p \in M \text { such that } X(p)=0,
$$

where $X$ is a locally Lipschitz continuous vector field on $M$. We will study the local and semi-local properties of the sequence generated by the method. In the following, we formally state the Newton method to solve the problem (4).

\section{Algorithm 1. Newton method}

Step 0. Let $p_{0} \in M$ be given, and set $k=0$.

Step 1. If $X\left(p_{k}\right)=0$, stop.

Step 2. Choose a $V_{k} \in \partial X\left(p_{k}\right)$ and compute

$$
p_{k+1}=\exp _{p_{k}}\left(-V_{k}^{-1} X\left(p_{k}\right)\right)
$$

Step 3. Set $k \leftarrow k+1$, and go to step 1 .

This method is a natural extension to the Riemannian setting of Newton method introduced in 31. Note that for to guarantee the well-definedness of the method, there are two issue which deserve attention in each iteration $k$. The Clarke generalized covariant derivative $\partial X\left(p_{k}\right)$ must be nonempty, which has already been proven in the item $(i)$ of Proposition 12 and $V_{k} \in \partial X\left(p_{k}\right)$ must be non-singular. In the following section, we will study the well-definedness and convergence properties of Newton method. 


\subsection{Local Convergence Analysis}

In this section, we present the local convergence analysis of Algorithm 1 For this end, we assume that $p_{*} \in M$ is a solution of problem (44). First, we will show that under some assumptions, the sequence generated by this algorithm starting from a suitable neighborhood of $p_{*}$ is well-defined and converges to $p_{*}$ with rate of order $1+\mu$. We begin by introducing the concept of regularity.

Definition 13. We say that a vector field $X$ on $M$ is regular at $p \in M$ if all $V_{p} \in \partial X(p)$ are non-singular. If $X$ is regular at every point of $\Omega \subseteq M$, we say that $X$ is regular on $\Omega$.

In the following, we study the behavior of the Newton method for a special class of vector field in a neighborhood of a regular point. For this, assume that $X$ is a locally Lipschitz continuous vector field on $M$. Consider the following condition:

A1. Let $\bar{p} \in M, 0<\delta<r_{\bar{p}}, X$ be regular on $B_{\delta}(\bar{p}), \lambda_{\bar{p}} \geq \max \left\{\left\|V_{\bar{p}}^{-1}\right\|: V_{\bar{p}} \in \partial X(\bar{p})\right\}$ and $\epsilon>0$ with $\epsilon \lambda_{\bar{p}}<1$. For all $p, q \in B_{\delta}(\bar{p})$ and $V_{p} \in \partial X(p)$ there hold

$$
\begin{aligned}
\left\|V_{p}^{-1}\right\| & \leq \frac{\lambda_{\bar{p}}}{1-\epsilon \lambda_{\bar{p}}} \\
\left\|X(q)-P_{p q}\left[X(p)+V_{p} \exp _{p}^{-1} q\right]\right\| & \leq \epsilon d(p, q)^{1+\mu}, \quad 0 \leq \mu \leq 1 .
\end{aligned}
$$

The above assumption guarantee, in particular, that $X$ is regular in a neighborhood of $\bar{p}$ and consequently, the Newton iteration mapping is well-defined. Let $0<\delta<r_{\bar{p}}$ be given by above assumption and $N_{X}: B_{\delta}(\bar{p}) \rightrightarrows M$ be the Newton iteration mapping for $X$ defined by

$$
N_{X}(p):=\left\{\exp _{p}\left(-V_{p}^{-1} X(p)\right): V_{p} \in \partial X(p)\right\} .
$$

Therefore, one can apply a single Newton iteration on any $p \in B_{\delta}(\bar{p})$ to obtain $N_{X}(p)$, which may be not included in $B_{\delta}(\bar{p})$. Thus, this is enough to guarantee the well-definedness of only one iteration. In the next result, we establish that Newtonian iterations may be repeated indefinitely in a suitable neighbourhood of $\bar{p}$.

Lemma 14. Suppose that $p_{*} \in M$ is a solution of problem (41), $X$ satisfies A1 with $\bar{p}=p_{*}, q=p_{*}$ and the constants $\epsilon>0,0<\delta<r_{p_{*}}$ and $0 \leq \mu \leq 1$ satisfy $\epsilon \lambda_{p_{*}}\left(1+\delta^{\mu} K_{p_{*}}\right)<1$. Then, there exists $\hat{\delta}>0$ such that $X$ is regular on $B_{\hat{\delta}}\left(p_{*}\right)$ and

$$
d\left(\exp _{p}\left(-V_{p}^{-1} X(p)\right), p_{*}\right) \leq \frac{\epsilon \lambda_{p_{*}} K_{p_{*}}}{1-\epsilon \lambda_{p_{*}}} d\left(p, p_{*}\right)^{1+\mu}, \quad \forall p \in B_{\hat{\delta}}\left(p_{*}\right), \quad \forall V_{p} \in \partial X(p) .
$$

Consequently, $N_{X}$ is well-defined on $B_{\hat{\delta}}\left(p_{*}\right)$ and $N_{X}(p) \subset B_{\hat{\delta}}\left(p_{*}\right)$ for all $p \in B_{\hat{\delta}}\left(p_{*}\right)$.

Proof. Since $\epsilon>0,0<\delta<r_{p_{*}}$ and $0 \leq \mu \leq 1$ are constants such that $X$ satisfies A1, $X\left(p_{*}\right)=0$ and the parallel transport is an isometry, we conclude that

$$
\begin{aligned}
\left\|V_{p}^{-1} X(p)+\exp _{p}^{-1} p_{*}\right\| & \leq\left\|V_{p}^{-1}\right\|\left\|X\left(p_{*}\right)-P_{p p_{*}}\left[X(p)+V_{p} \exp _{p}^{-1} p_{*}\right]\right\| \\
& \leq \frac{\epsilon \lambda_{p_{*}}}{1-\epsilon \lambda_{p_{*}}} d\left(p, p_{*}\right)^{1+\mu}
\end{aligned}
$$

for all $p \in B_{\delta}\left(p_{*}\right)$ and $V_{p} \in \partial X(p)$. Hence, (9) implies that there exists $0<\hat{\delta}<\delta$ such that $\left\|V_{p}^{-1} X(p)+\exp _{p}^{-1} p_{*}\right\| \leq r_{p_{*}}$ for all $p \in B_{\hat{\delta}}\left(p_{*}\right)$ and $V_{p} \in \partial X(p)$. Thus, considering that 
$\left\|\exp _{p}^{-1} p_{*}\right\|=d\left(p, p_{*}\right)<r_{p_{*}}$, we can use Definition 2 with $p=p_{*}, q=p, u=-V_{p}^{-1} X(p)$ and $v=\exp _{p}^{-1} p_{*}$ to obtain that

$$
d\left(\exp _{p}\left(-V_{p}^{-1} X(p)\right), p_{*}\right) \leq K_{p_{*}}\left\|-V_{p}^{-1} X(p)-\exp _{p}^{-1} p_{*}\right\|,
$$

for all $p \in B_{\hat{\delta}}\left(p_{*}\right)$ and $V_{p} \in \partial X(p)$. Therefore, the combination of the last inequality with (9) yields (8). Owing to $0<\hat{\delta}<\delta$ and $X$ be regular on $B_{\delta}\left(p_{*}\right)$, we conclude that $N_{X}$ is welldefined on $B_{\hat{\delta}}\left(p_{*}\right)$. Moreover, since $\epsilon \lambda_{p_{*}}\left(1+\delta^{\mu} K_{p_{*}}\right)<1$ and $0<\hat{\delta}<\delta$ we have from (8) that $d\left(\exp _{p}\left(-V_{p}^{-1} X(p)\right), p_{*}\right)<d\left(p, p_{*}\right)$ for all $p \in B_{\hat{\delta}}\left(p_{*}\right)$ and $V_{p} \in \partial X(p)$. Thus, we obtain that $N_{X}(p) \subset B_{\hat{\delta}}\left(p_{*}\right)$ for all $p \in B_{\hat{\delta}}\left(p_{*}\right)$, and the proof of the lemma is complete.

Now, we are ready to establishes the main result of this section, its prove is a straight application of Lemma 14.

Theorem 15. Suppose that $p_{*} \in M$ is a solution of problem (4), $X$ satisfies A1 with $\bar{p}=p_{*}$, $q=p_{*}$ and the constants $\epsilon>0,0<\delta<r_{p_{*}}$ and $0 \leq \mu \leq 1$ satisfy $\epsilon \lambda_{p_{*}}\left(1+\delta^{\mu} K_{p_{*}}\right)<1$. Then, there exists $0<\hat{\delta}<\delta$ such that for each $p_{0} \in B_{\hat{\delta}}\left(p_{*}\right) \backslash\left\{p_{*}\right\},\left\{p_{k}\right\}$ in Algorithm 1 is well-defined, belongs to $B_{\hat{\delta}}\left(p_{*}\right)$ and converges to $p_{*}$ with order $1+\mu$ as follows

$$
d\left(p_{k+1}, p_{*}\right) \leq \frac{\epsilon \lambda_{p_{*}} K_{p_{*}}}{1-\epsilon \lambda_{p_{*}}} d\left(p_{k}, p_{*}\right)^{1+\mu}, \quad k=0,1, \ldots
$$

Proof. The definition of Newton mapping $N_{X}$ implies that the sequence generated by Algorithm 1 is equivalently stated as

$$
p_{k+1} \in N_{X}\left(p_{k}\right), \quad k=0,1, \ldots
$$

Thus, by using (11), we can apply Lemma 14 to conclude that there exists $0<\hat{\delta}<\delta$ such that if $p_{0} \in B_{\hat{\delta}}\left(p_{*}\right) \backslash\left\{p_{*}\right\}$, then $\left\{p_{k}\right\}$ in Algorithm 1 is well-defined, belongs to $B_{\hat{\delta}}\left(p_{*}\right)$ and satisfies (10). Since $\left\{p_{k}\right\}$ belongs to $B_{\hat{\delta}}\left(p_{*}\right)$ and $\epsilon \lambda_{p_{*}}\left(1+\delta^{\mu} K_{p_{*}}\right)<1$ we obtain from (10) that

$$
d\left(p_{k+1}, p_{*}\right)<\frac{\epsilon \lambda_{p_{*}} \hat{\delta}^{\mu} K_{p_{*}}}{1-\epsilon \lambda_{p_{*}}} d\left(p_{k}, p_{*}\right)<d\left(p_{k}, p_{*}\right), \quad k=0,1, \ldots
$$

Therefore, we conclude that $\left\{p_{k}\right\}$ converges to $p_{*}$ with order $1+\mu$ as (10).

Remark 16. If $\mu=0$ in Theorem 15, then (10) holds for any $\epsilon>0$ satisfying $\epsilon \lambda_{p_{*}}\left(1+K_{p_{*}}\right)<1$, independently of $\hat{\delta}$. Thus, (10) implies that $\left\{p_{k}\right\}$ converges superlinearly to $p_{*}$.

\subsubsection{Local Convergence for Semismooth Vector Fields}

In this section, we present a local convergence theorem for the Newton method for finding a singularity of semismooth vector fields. Semismoothness in Euclidean setting was originally introduced by Mifflin [29] for scalar-valued functions and subsequently extended by Qi and Sun in [31] for vector-valued functions. The extension of semismoothness to the Riemannian settings was presented in [24], and it will plays an important role in this section. As occur in Euclidean context, semismooth vector fields are in general nonsmooth. However, as we shall show the Newton method is still applicable and converges locally with superlinear rate to a regular solution. Before state formally the concept of semismoothness in the Riemannian setting, let us first to show that locally Lipschitz continuous vector fields are regular near regular points. The statement of the result is: 
Lemma 17. Let $X$ be a locally Lipschitz continuous vector field on $M$. Assume that $X$ is regular at $p_{*} \in M$ and let $\lambda_{p_{*}} \geq \max \left\{\left\|V_{p_{*}}^{-1}\right\|: V_{p_{*}} \in \partial X\left(p_{*}\right)\right\}$. Then, for every $\epsilon>0$ satisfying $\epsilon \lambda_{p_{*}}<1$, there exists $0<\delta<r_{p_{*}}$ such that $X$ is regular on $B_{\delta}\left(p_{*}\right)$ and

$$
\left\|V_{p}^{-1}\right\| \leq \frac{\lambda_{p_{*}}}{1-\epsilon \lambda_{p_{*}}}, \quad \forall p \in B_{\delta}\left(p_{*}\right), \quad \forall V_{p} \in \partial X(p) .
$$

Proof. Let $\epsilon>0$ such that $\epsilon \lambda_{p_{*}}<1$. Since $X$ is a locally Lipschitz continuous vector field, it follows from item (iii) of Proposition 12 that, there is a $0<\delta<r_{p_{*}}$ such that for all $p \in B_{\delta}\left(p_{*}\right)$, $P_{p p_{*}} \partial X(p) \subset \partial X\left(p_{*}\right)+\left\{V \in T_{p_{*}} M:\|V\|<\epsilon\right\}$, i.e.,

$$
\partial X(p) \subset\left\{V \in T_{p} M:\left\|P_{p p_{*}} V-V_{p_{*}}\right\|<\epsilon, \text { for some } V_{p_{*}} \in \partial X\left(p_{*}\right)\right\}, \quad \forall p \in B_{\delta}\left(p_{*}\right) .
$$

This inclusion implies that, for each $p \in B_{\delta}\left(p_{*}\right)$ and $V_{p} \in \partial X(p)$, there exists $V_{p_{*}} \in \partial X\left(p_{*}\right)$ nonsingular such that $\left\|V_{p_{*}}^{-1}\right\|\left\|P_{p_{*}} V_{p}-V_{p_{*}}\right\|<\epsilon \lambda_{p_{*}}<1$. Thus, taking into account that the parallel transport is an isometry, it follows from Lemma 5 that $V_{p}$ is non-singular and

$$
\left\|V_{p}^{-1}\right\| \leq \frac{\left\|V_{p_{*}}^{-1}\right\|}{1-\left\|V_{p_{*}}^{-1}\right\|\left\|P_{p p_{*}} V_{p}-V_{p_{*}}\right\|} .
$$

Therefore, considering that $\left\|V_{p_{*}}^{-1}\right\| \leq \lambda_{p_{*}}$ and $\left\|P_{p p_{*}} V_{p}-V_{p_{*}}\right\|<\epsilon$, the inequality (12) follows.

Now, let us present a class of vector fields satisfying the assumption A1, namely the semismooth vector fields and $\mu$-order semismooth vector fields. There exist, in the Euclidean context, several equivalents definitions of the concept of semismoothness, see [31, see also 12, Definition 7.4 .2 , p. 677]. In the present paper, we will extend to the Riemannian settings the concept of semismoothness adopted in [10, p. 411].

Definition 18. A vector field $X$ on $M$, which is Lipschitz continuous at $p_{*}$ and directionally differentiable at $p \in B_{\delta}\left(p_{*}\right)$ for all direction at $T_{p} M$, is said to be semismooth at $p_{*} \in M$ when for every $\epsilon>0$ there exists $0<\delta<r_{p_{*}}$ such that

$$
\left\|X\left(p_{*}\right)-P_{p p_{*}}\left[X(p)+V_{p} \exp _{p}^{-1} p_{*}\right]\right\| \leq \epsilon d\left(p, p_{*}\right), \quad \forall p \in B_{\delta}\left(p_{*}\right), \quad \forall V_{p} \in \partial X(p) .
$$

The vector filed $X$ is said to be $\mu$-order semismooth at $p_{*} \in M$, for $0<\mu \leq 1$, when there exist $\epsilon>0$ and $0<\delta<r_{p_{*}}$ such that

$$
\left\|X\left(p_{*}\right)-P_{p p_{*}}\left[X(p)+V_{p} \exp _{p}^{-1} p_{*}\right]\right\| \leq \epsilon d\left(p, p_{*}\right)^{1+\mu}, \quad \forall p \in B_{\delta}\left(p_{*}\right), \quad \forall V_{p} \in \partial X(p) .
$$

Next, we state and prove the local convergence result for the Newton method for finding a singularity of semismooth and $\mu$-order semismooth vector fields.

Theorem 19. Let $X$ be locally Lipschitz continuous vector filed on $M$ and $p_{*} \in M$ be a solution of problem (4). Assume that $X$ is semismooth and regular at $p_{*}$. Then, there exists a $\delta>0$ such that for each $p_{0} \in B_{\delta}\left(p_{*}\right) \backslash\left\{p_{*}\right\},\left\{p_{k}\right\}$ generated by Algorithm 1, is well-defined, belongs to $B_{\delta}\left(p_{*}\right)$ and converges superlinearly to $p_{*}$. Additionally, if $X$ is $\mu$-order semismooth at $p_{*}$, then the convergence of $\left\{p_{k}\right\}$ to $p_{*}$ is of order $1+\mu$.

Proof. Owing to $X$ be semismooth and regular at $p_{*} \in M$, we can take $\lambda_{p_{*}} \geq \max \left\{\left\|V_{p_{*}}^{-1}\right\|: V_{p_{*}} \in\right.$ $\left.\partial X\left(p_{*}\right)\right\}$. Take $\epsilon>0$ satisfying $\epsilon \lambda_{p_{*}}\left(1+K_{p_{*}}\right)<1$. Thus, from Lemma 17 and Definition 18 we can take $\delta>0$ such that (6) and (7) hold for $\mu=0$. Hence, assumption A1 holds with $\bar{p}=p_{*}$ and 
$q=p_{*}$ for all $p \in B_{\delta}\left(p_{*}\right)$ and $\mu=0$. Therefore, applying Theorem [15, we obtain that there exists $0<\hat{\delta}<\delta$ such that every sequence $\left\{p_{k}\right\}$ generated by Algorithm 1 with $p_{0} \in B_{\hat{\delta}}\left(p_{*}\right) \backslash\left\{p_{*}\right\}$ belongs to $B_{\hat{\delta}}\left(p_{*}\right)$ and satisfies (10). Hence, we have

$$
\frac{d\left(p_{k+1}, p_{*}\right)}{d\left(p_{k}, p_{*}\right)} \leq \frac{\epsilon \lambda_{p_{*}} K_{p_{*}}}{1-\epsilon \lambda_{p_{*}}}, \quad k=0,1, \ldots
$$

Since the last equality holds for any $\epsilon$ such that $0<\epsilon<1 /\left(\lambda_{p_{*}}\left(1+K_{p_{*}}\right)\right)$, we conclude that $\left\{p_{k}\right\}$ converges superlinearly to $p_{*}$. The proof of the second part is similar. Indeed, for a given $\epsilon>0$ with $\epsilon \lambda_{p_{*}}<1$, take $\delta>0$ satisfying $\epsilon \lambda_{p_{*}}\left(1+\delta^{\mu} K_{p_{*}}\right)<1$ and such that (12) and (13) hold. Then, we can apply Theorem 15 and the proof follows.

In the following remark, in particular, we show that with some adjustments Theorem 19 reduces to some well-known results.

Remark 20. It is well-known that Newton method and its variants are quite efficient for finding zero on nonlinear functions in Euclidean settings. This because they have excellent convergence rate in a neighborhood of a zero. It was shown in [31] that for some class of nonsmooth functions, namely for semismooth functions, the convergence of Newton method still is guaranteed. The above theorem, allows us conclude that the generalization of Newton method from the linear context to Riemannian settings for finding singularities of semismooth vector fields still preserves its main convergence properties. It is worth mentioning that if $X$ is continuously differentiable, then Theorem 19 reduces to [13, Theorem 3.1]. If $M=\mathbb{R}^{n}$, then Theorem 19 reduces to first part of [31, Theorem 3.2], see

also [12, Theorem 7.5.3, p. 693]. Finally, if $X$ is continuously differentiable and $M=\mathbb{R}^{n}$, then the theorem above reduces to the first part of [4, Proposition 1.4.1, p. 90].

\subsection{Semi-local Convergence Analysis}

In this section, we state and prove the Kantorovich-type theorem on Newton method. This theorem ensures that the sequence generated by the method converges towards a singularity of the vector field by using semi-local conditions. It is worth noting that the theorem does not require a priori existence of a singularity, proving instead the existence of the singularity and its uniqueness on some region. The statement of the theorem is:

Theorem 21. Let $X$ be locally Lipschitz continuous vector filed on $M$ and $p_{0} \in M$. Suppose that $X$ satisfies $\mathbf{A} 1$ with $\bar{p}=p_{0}, \mu=0$ and $\delta>\bar{\delta}$. Moreover, $B_{\bar{\delta}}\left(p_{0}\right) \subset M$ is a totally normal neighborhood of $p_{0}$ and the constants $\lambda_{p_{0}}>0, \epsilon>0$ and $0<\bar{\delta}<r_{p_{0}}$ are such that

$$
\epsilon \lambda_{p_{0}}<\frac{1}{2}, \quad \frac{\lambda_{p_{0}}}{1-2 \epsilon \lambda_{p_{0}}}\left\|X\left(p_{0}\right)\right\| \leq \bar{\delta} .
$$

Then, $\left\{p_{k}\right\}$ in Algorithm 1 is well-defined, belongs to $B_{\bar{\delta}}\left(p_{0}\right)$ and converge towards the unique solution $p_{*}$ of problem (4) in $B_{\bar{\delta}}\left[p_{0}\right]$. Furthermore, the following error estimate holds

$$
d\left(p_{k}, p_{*}\right) \leq \frac{\epsilon \lambda_{p_{0}}}{1-2 \epsilon \lambda_{p_{0}}} d\left(p_{k}, p_{k-1}\right), \quad k=1,2, \ldots
$$

Proof. Firstly, we will prove by induction that the sequence $\left\{p_{k}\right\}$ in Algorithm 1 is well-defined, belong to $B_{\bar{\delta}}\left(p_{0}\right)$ and satisfies

$$
d\left(p_{k+1}, p_{k}\right) \leq\left(\frac{\epsilon \lambda_{p_{0}}}{1-\epsilon \lambda_{p_{0}}}\right)^{k} \bar{\delta}\left(\frac{1-2 \epsilon \lambda_{p_{0}}}{1-\epsilon \lambda_{p_{0}}}\right), \quad k=0,1, \ldots
$$


Let $V_{0} \in \partial X\left(p_{0}\right)$. Assumption $\mathbf{A} 1$ implies that $V_{0}$ is non-singular and $\left\|V_{0}^{-1}\right\| \leq \lambda_{p_{0}} /\left(1-\epsilon \lambda_{p_{0}}\right)$. Hence using (5) we obtain that the iterate $p_{1}$ is well-defined. Furthermore, the definition of the exponential mapping and (14) imply that

$$
d\left(p_{1}, p_{0}\right)=d\left(\exp _{p_{0}}\left(-V_{0}^{-1} X\left(p_{0}\right)\right), p_{0}\right) \leq\left\|-V_{0}^{-1} X\left(p_{0}\right)\right\| \leq \frac{\lambda_{p_{0}}}{1-\epsilon \lambda_{p_{0}}}\left\|X\left(p_{0}\right)\right\| \leq \bar{\delta}\left(\frac{1-2 \epsilon \lambda_{p_{0}}}{1-\epsilon \lambda_{p_{0}}}\right)<\bar{\delta} .
$$

Therefore, $p_{1}$ is well-defined, belong to $B_{\bar{\delta}}\left(p_{0}\right)$ and (16) holds for $k=0$. Assume by induction that $p_{1}, \ldots, p_{\ell-1}$ are well-defined, belong to $B_{\bar{\delta}}\left(p_{0}\right)$ and (16) holds for $k=1, \ldots, \ell-1$. Since $p_{\ell-1} \in B_{\bar{\delta}}\left(p_{0}\right)$ it follows from assumption $\mathbf{A} 1$ that $V_{\ell-1}$ is non-singular and, in particular, the iterate $p_{\ell}$ is well-defined. Thus, using triangular inequality and the induction assumption we have

$$
d\left(p_{\ell}, p_{0}\right) \leq \sum_{j=1}^{\ell} d\left(p_{j}, p_{j-1}\right) \leq \bar{\delta}\left(\frac{1-2 \epsilon \lambda_{p_{0}}}{1-\epsilon \lambda_{p_{0}}}\right) \sum_{j=1}^{\ell}\left(\frac{\epsilon \lambda_{p_{0}}}{1-\epsilon \lambda_{p_{0}}}\right)^{j-1}<\bar{\delta}
$$

Then, $p_{\ell} \in B_{\bar{\delta}}\left(p_{0}\right)$. Since $p_{\ell} \in B_{\bar{\delta}}\left(p_{0}\right)$ it follows from A1 that $V_{\ell}$ is non-singular and, in particular, the iterate $p_{\ell+1}$ is well-defined. Moreover, $\left\|V_{\ell}^{-1}\right\| \leq \lambda_{p_{0}} /\left(1-\epsilon \lambda_{p_{0}}\right)$. Thus, using (5) and the definition of the exponential mapping we have

$$
d\left(p_{\ell+1}, p_{\ell}\right)=d\left(\exp _{p_{\ell}}\left(-V_{\ell}^{-1} X\left(p_{\ell}\right)\right), p_{\ell}\right) \leq\left\|-V_{\ell}^{-1} X\left(p_{\ell}\right)\right\| \leq \frac{\lambda_{p_{0}}}{1-\epsilon \lambda_{p_{0}}}\left\|X\left(p_{\ell}\right)\right\| .
$$

On the other hand, considering that $B_{\bar{\delta}}\left(p_{0}\right)$ is a totally normal neighborhood and $p_{\ell-1}, p_{\ell} \in B_{\bar{\delta}}\left(p_{0}\right)$, we conclude after some algebraic manipulations that

$$
\left\|X\left(p_{\ell}\right)\right\| \leq\left\|X\left(p_{\ell}\right)-P_{p_{\ell-1} p_{\ell}}\left[X\left(p_{\ell-1}\right)+V_{\ell-1} \exp _{p_{\ell-1}}^{-1} p_{\ell}\right]\right\|+\left\|X\left(p_{\ell-1}\right)+V_{\ell-1} \exp _{p_{\ell-1}}^{-1} p_{\ell}\right\| .
$$

Taking into account that (5) implies $X\left(p_{\ell-1}\right)+V_{\ell-1} \exp _{p_{\ell-1}}^{-1} p_{\ell}=0$ the last inequality becomes

$$
\left\|X\left(p_{\ell}\right)\right\| \leq\left\|X\left(p_{\ell}\right)-P_{p_{\ell-1} p_{\ell}}\left[X\left(p_{\ell-1}\right)+V_{\ell-1} \exp _{p_{\ell-1}}^{-1} p_{\ell}\right]\right\| .
$$

Using A1 with $q=p_{\ell}, p=p_{\ell-1}$ and $V_{p}=V_{\ell-1}$, it follows from the latter inequality that

$$
\left\|X\left(p_{\ell}\right)\right\| \leq \epsilon d\left(p_{\ell}, p_{\ell-1}\right) .
$$

Thus combining the last inequality with (18) and using the induction assumption we conclude that

$$
d\left(p_{\ell+1}, p_{\ell}\right) \leq \frac{\epsilon \lambda_{p_{0}}}{1-\epsilon \lambda_{p_{0}}} d\left(p_{\ell}, p_{\ell-1}\right) \leq\left(\frac{\epsilon \lambda_{p_{0}}}{1-\epsilon \lambda_{p_{0}}}\right)^{\ell} \bar{\delta}\left(\frac{1-2 \epsilon \lambda_{p_{0}}}{1-\epsilon \lambda_{p_{0}}}\right)
$$

and the induction proof is complete. Hence, using (19) and the same argument used to prove (17) we obtain that $p_{\ell+1} \in B_{\bar{\delta}}\left(p_{0}\right)$. Therefore, the Newton iterates are well-defined, belong to $B_{\bar{\delta}}\left(p_{0}\right)$ and satisfy (16). We proceed to prove that $\left\{p_{k}\right\}$ converges. Using the triangular inequality and (16), for any $k$ and $s \in\{0,1, \ldots\}$ we have

$$
d\left(p_{k+s+1}, p_{k}\right) \leq \sum_{j=k}^{k+s} d\left(p_{j+1}, p_{j}\right) \leq \bar{\delta}\left(\frac{1-2 \epsilon \lambda_{p_{0}}}{1-\epsilon \lambda_{p_{0}}}\right) \sum_{j=k}^{k+s}\left(\frac{\epsilon \lambda_{p_{0}}}{1-\epsilon \lambda_{p_{0}}}\right)^{j}<\bar{\delta}\left(\frac{\epsilon \lambda_{p_{0}}}{1-\epsilon \lambda_{p_{0}}}\right)^{k} .
$$

Thus, due to $2 \epsilon \lambda_{p_{0}}<1$ we conclude that $\left\{p_{k}\right\}$ is a Cauchy sequence. This implies that the sequence $\left\{p_{k}\right\}$ converges to some $p_{*} \in B_{\bar{\delta}}\left[p_{0}\right]$. Thus, owing to $X$ be a locally Lipschitz continuous vector field, 
item (ii) of Proposition 12 implies that $\left\{V_{k}\right\}$ is bounded. Therefore, using that $X$ is continuous, (5), some properties of norm and $\left\{p_{k}\right\}$ converges to $p_{*}$, we have

$$
0 \leq\left\|X\left(p_{*}\right)\right\|=\lim _{k \rightarrow+\infty}\left\|X\left(p_{k}\right)\right\|=\lim _{k \rightarrow+\infty}\left\|-V_{k} \exp _{p_{k}}^{-1} p_{k+1}\right\| \leq \lim _{k \rightarrow+\infty}\left\|V_{k}\right\| d\left(p_{k+1}, p_{k}\right)=0
$$

consequently, $X\left(p_{*}\right)=0$. Now, we are going to prove the uniqueness of the solution in $B_{\bar{\delta}}\left[p_{0}\right]$. For this purpose, assume that $q \in B_{\bar{\delta}}\left[p_{0}\right]$ and $X(q)=0$. Take $V_{*} \in \partial X\left(p_{*}\right)$, by assumption $X$ is regular on $B_{\delta}\left(p_{0}\right)$ and $B_{\bar{\delta}}\left[p_{0}\right] \subset B_{\delta}\left(p_{0}\right)$, then $V_{*}$ is non-singular. Since $X\left(p_{*}\right)=0$ and $X(q)=0$, using $\mathbf{A} 1$ with $p=p_{*}$ and $V_{p}=V_{*}$, and some manipulations we obtain that

$$
d\left(p_{*}, q\right)=\left\|V_{*}^{-1} V_{*} \exp _{p_{*}}^{-1} q\right\| \leq\left\|V_{*}^{-1}\right\|\left[\left\|X(q)-P_{p_{*} q}\left[X\left(p_{*}\right)+V_{*} \exp _{p_{*}}^{-1} q\right]\right\|\right] \leq \frac{\epsilon \lambda_{p_{0}}}{1-\epsilon \lambda_{p_{0}}} d\left(p_{*}, q\right) .
$$

Thus, since $\epsilon \lambda_{p_{0}}<1 / 2$ we conclude that $d\left(p_{*}, q\right)=0$, i.e., $q=p_{*}$. Therefore, $p_{*}$ is the unique solution of (4) in $B_{\bar{\delta}}\left[p_{0}\right]$. It remains to show (15). First note that using the same argument to establishes first inequality in (19) we can also prove that $d\left(p_{i+1}, p_{i}\right) \leq\left[\epsilon \lambda_{p_{0}} /\left(1-\epsilon \lambda_{p_{0}}\right)\right] d\left(p_{i}, p_{i-1}\right)$, for all $i=1,2, \ldots$ and then

$$
d\left(p_{k+j}, p_{k+j-1}\right) \leq\left(\frac{\epsilon \lambda_{p_{0}}}{1-\epsilon \lambda_{p_{0}}}\right)^{j} d\left(p_{k}, p_{k-1}\right), \quad j=1,2, \ldots
$$

Hence, using the triangular inequality, the last inequality and any $s \in\{0,1, \ldots\}$ we conclude

$$
d\left(p_{k+s+1}, p_{k}\right) \leq \sum_{j=k}^{k+s} d\left(p_{j+1}, p_{j}\right) \leq d\left(p_{k}, p_{k-1}\right) \sum_{j=1}^{s+1}\left(\frac{\epsilon \lambda_{p_{0}}}{1-\epsilon \lambda_{p_{0}}}\right)^{j}<\frac{\epsilon \lambda_{p_{0}}}{1-2 \epsilon \lambda_{p_{0}}} d\left(p_{k}, p_{k-1}\right) .
$$

Taking the limit as $s$ goes to $+\infty$, we obtain the inequality (15), and the proof is complete.

\section{$5 \quad$ Some Examples}

In this section, we present a class of examples of locally Lipschitz continuous vector fields on the sphere satisfying the assumption A1. For this purpose, we begin by presenting some basic definitions about the geometry of the sphere. For further details, see [14, 15] and references therein.

Let $\langle\cdot, \cdot\rangle$ be the usual inner product on $\mathbb{R}^{n+1}$, with corresponding norm denoted by $\|\cdot\|$. The $n$-dimensional Euclidean sphere and its tangent hyperplane at a point $p$ are denoted, respectively, by

$$
\mathbb{S}^{n}:=\left\{p=\left(p_{1}, \ldots, p_{n+1}\right) \in \mathbb{R}^{n+1}:\|p\|=1\right\}, \quad T_{p} \mathbb{S}^{n}:=\left\{v \in \mathbb{R}^{n+1}:\langle p, v\rangle=0\right\} .
$$

Denotes by $I$ the $(n+1) \times(n+1)$ identity matrix. The projection onto the tangent hyperplane $T_{p} \mathbb{S}^{n}$ is the linear mapping defined by $I-p p^{T}: \mathbb{R}^{n+1} \rightarrow T_{p} \mathbb{S}^{n}$, where $p^{T}$ denotes the transpose of the vector $p$. Let $\Omega$ be an open set in $\mathbb{R}^{n+1}$ such that $\mathbb{S}^{n} \subset \Omega$, and $Y: \Omega \rightarrow \mathbb{R}^{n+1}$ be any semismooth mapping; several examples can be found in [10, 12, 23]. Then, we define the vector field $X: \mathbb{S}^{n} \rightarrow \mathbb{R}^{n+1}$ as follows

$$
X(p):=\left(I-p p^{T}\right) Y(p) .
$$

Note that $X(p) \in T_{p} \mathbb{S}^{n}$ for all $p \in \mathbb{S}^{n}$. The Clarke generalized covariant derivative of $X$ at $p$ is given by

$$
\partial X(p):=\left(I-p p^{T}\right) \partial Y(p)-p^{T} Y(p) I,
$$


where $\partial Y(p)$ is the Clarke generalized covariant derivative of $Y$ at $p$. Therefore, all $V_{p} \in \partial X(p)$ is a linear mapping $V_{p}: T_{p} \mathbb{S}^{n} \rightarrow T_{p} \mathbb{S}^{n}$ given by $V_{p}:=\left(I-p p^{T}\right) \tilde{V}_{p}-p^{T} Y(p) I$, where $\tilde{V}_{p} \in \partial Y(p)$. Since $Y$ is a locally Lipschitz continuous mapping, from Rademacher theorem, see [11, Theorem 2, p. 81], we conclude that $Y$ is almost everywhere differentiable. As $I-p p^{T}$ is a differentiable mapping, we obtain that $X$ is almost everywhere differentiable. By using the fundamental theorem of calculus in Riemannian setting (see [16]), the fact that $\partial Y(p)$ is locally bounded and continuity of $Y$, we can prove that $X$ is also locally Lipschitz continuous vector field. Assume that $X$ is regular at $\bar{p} \in \Omega$ and let $\lambda_{\bar{p}} \geq \max \left\{\left\|V_{\bar{p}}^{-1}\right\|: V_{\bar{p}} \in \partial X(\bar{p})\right\}$. Then, from Lemma 17 for every $\epsilon>0$ satisfying $\epsilon \lambda_{\bar{p}}<1$, there exists $0<\delta<\pi$ (where $\pi$ is the injectivity radius of $\mathbb{S}^{n}$ ) such that $X$ is regular on $B_{\delta}(\bar{p})$ and for all $p \in B_{\delta}(\bar{p})$ and $V_{p} \in \partial X(p)$ the following holds

$$
\left\|V_{p}^{-1}\right\| \leq \frac{\lambda_{\bar{p}}}{1-\epsilon \lambda_{\bar{p}}}
$$

This implies that inequality (6) holds. On the other hand, because $X$ is a composition of semismooth mappings, we conclude that $X$ is semismooth, see [23, Proposition 1.74, p. 54]. Hence, from Definition 18 inequality (7) holds. Therefore, the projected vector field $X$ satisfies the assumption A1. In the following, we present a concrete example.

Example 22. Let $Y: \mathbb{R}^{2} \rightarrow \mathbb{R}^{2}$ be a semismooth mapping defined by $Y(p):=A p-|p|-b$ with matrix $A=\operatorname{diag}(4,3)$ and vector $b=\left(b_{1}, b_{2}\right) \in \mathbb{R}^{2}$, where diag $\left(p_{1}, p_{2}\right)$ denotes a $2 \times 2$ diagonal matrix with $(i, i)$-th entry equal to $p_{i}, i=1,2$. Take $\bar{p}=(0,1) \in \mathbb{S}^{2}$ and note that $Y(\bar{p})=0$ for $b=(0,2)$. Some calculus show that the Clarke generalized covariant derivative of $Y$ at $\bar{p}$ is given by $\partial Y(\bar{p})=\{\operatorname{diag}(d, 2): d \in[3,5]\}$. Define $X(p):=\left(I-p p^{T}\right) Y(p)$ the vector field on $\mathbb{S}^{2}$. Therefore, using (20), we conclude that $\partial X(\bar{p})=\left\{V_{\bar{p}}:=\operatorname{diag}\left(d-2+b_{2},-2+b_{2}\right): d \in[3,5]\right\}$. Note that all $V_{\bar{p}} \in \partial X(\bar{p})$ are non-singular as a linear mapping $V_{\bar{p}}: T_{\bar{p}} \mathbb{S}^{2} \rightarrow T_{\bar{p}} \mathbb{S}^{2}$, where the tangent hyperplane at $\bar{p}$ is given by $T_{\bar{p}} \mathbb{S}^{2}:=\left\{v:=\left(v_{1}, 0\right) \in \mathbb{R}^{2}: v_{1} \in \mathbb{R}\right\}$. Hence, from Definition 13 , we obtain that $X$ is regular at $\bar{p}=(0,1)$. Let $\lambda_{\bar{p}} \geq \max \left\{\left\|V_{\bar{p}}^{-1}\right\|: V_{\bar{p}} \in \partial X(\bar{p})\right\}$. As $X$ is a locally Lipschitz continuous vector field, using Lemma 17 for every $\epsilon>0$ satisfying $\epsilon \lambda_{\bar{p}}<1$, there exists $0<\delta<\pi$ such that $X$ is regular on $B_{\delta}(\bar{p})$ and for all $p \in B_{\delta}(\bar{p})$ and $V_{p} \in \partial X(p)$ the following hold $\left\|V_{p}{ }^{-1}\right\| \leq \lambda_{\bar{p}} /\left(1-\epsilon \lambda_{\bar{p}}\right)$. Because $X$ is a semismooth vector field, we conclude that the assumption A1 holds.

It is worth pointing out that in the literature there exist other examples of semismooth vector field, see, for example, 22].

\section{Conclusions}

In this paper, we studied the concept and some properties of the locally Lipschitz continuous vector fields. It is worth mentioning that the Rademacher theorem is an essential tool to ensure the existence of Clarke generalized covariant derivative. Additionally, a version of Newton method for finding a singularity these vector fields was proposed. Under regularity and semismoothness the well-definedness and local convergence of the method were established. Furthermore, a Kantorovichtype theorem was presented. We expect that the results of this paper can aid in the extensions of new results and methods of nonsmooth analysis to the Riemannian context, for example, the Mean Value Theorem as well as inexact and globalized versions of Newton method. 


\section{Acknowledgements}

The work was supported by CAPES, FAPEG and CNPq Grants 408151/2016-1, 302473/2017-3.

\section{References}

[1] D. Azagra, J. Ferrera, and F. López-Mesas. Nonsmooth analysis and Hamilton-Jacobi equations on Riemannian manifolds. J. Funct. Anal., 220(2):304-361, 2005.

[2] M. Bačák, R. Bergmann, G. Steidl, and A. Weinmann. A second order nonsmooth variational model for restoring manifold-valued images. SIAM J. Sci. Comput., 38(1):A567-A597, 2016.

[3] R. Bergmann and A. Weinmann. A second-order TV-type approach for inpainting and denoising higher dimensional combined cyclic and vector space data. J. Math. Imaging Vision, 55(3):401-427, 2016.

[4] D. P. Bertsekas. Nonlinear programming. Athena Scientific Optimization and Computation Series. Athena Scientific, Belmont, MA, third edition, 2016.

[5] R. D. Canary, D. Epstein, and A. Marden. Fundamentals of hyperbolic geometry: selected expositions, volume 328 of London Mathematical Society Lecture Note Series. Cambridge University Press, Cambridge, 2006.

[6] F. H. Clarke. Optimization and nonsmooth analysis, volume 5 of Classics in Applied Mathematics. Society for Industrial and Applied Mathematics (SIAM), Philadelphia, PA, second edition, 1990.

[7] J. X. da Cruz Neto, L. L. de Lima, and P. R. Oliveira. Geodesic algorithms in Riemannian geometry. Balkan J. Geom. Appl., 3(2):89-100, 1998.

[8] J.-P. Dedieu, P. Priouret, and G. Malajovich. Newton's method on Riemannian manifolds: convariant alpha theory. IMA J. Numer. Anal., 23(3):395-419, 2003.

[9] M. P. do Carmo. Riemannian geometry. Mathematics: Theory \& Applications. Birkhäuser Boston, Inc., Boston, MA, 1992. Translated from the second Portuguese edition by Francis Flaherty.

[10] A. L. Dontchev and R. T. Rockafellar. Implicit functions and solution mappings. Springer Series in Operations Research and Financial Engineering. Springer, New York, second edition, 2014. A view from variational analysis.

[11] L. C. Evans and R. F. Gariepy. Measure theory and fine properties of functions. Studies in Advanced Mathematics. CRC Press, Boca Raton, FL, 1992.

[12] F. Facchinei and J.-S. Pang. Finite-dimensional variational inequalities and complementarity problems. Vol. II. Springer Series in Operations Research. Springer-Verlag, New York, 2003.

[13] T. A. Fernandes, O. P. Ferreira, and J. Yuan. On the Superlinear Convergence of Newton's Method on Riemannian Manifolds. J. Optim. Theory Appl., 173(3):828-843, 2017.

[14] O. P. Ferreira, A. N. Iusem, and S. Z. Németh. Projections onto convex sets on the sphere. J. Optim. Theory Appl., 57(3):663-676, 2013. 
[15] O. P. Ferreira, A. N. Iusem, and S. Z. Németh. Concepts and techniques of optimization on the sphere. TOP, 22(3):1148-1170, 2014.

[16] O. P. Ferreira and B. F. Svaiter. Kantorovich's theorem on Newton's method in Riemannian manifolds. J. Complexity, 18(1):304-329, 2002.

[17] D. Gabay. Minimizing a differentiable function over a differential manifold. J. Optim. Theory Appl., 37(2):177-219, 1982.

[18] E. Ghahraei, S. Hosseini, and M. R. Pouryayevali. Pseudo-Jacobian and characterization of monotone vector fields on Riemannian manifolds. J. Convex Anal., 24(1):149-168, 2017.

[19] P. Grohs and S. Hosseini. Nonsmooth trust region algorithms for locally Lipschitz functions on Riemannian manifolds. IMA J. Numer. Anal., 36(3):1167-1192, 2016.

[20] S. Hosseini and M. R. Pouryayevali. Generalized gradients and characterization of epi-Lipschitz sets in Riemannian manifolds. Nonlinear Anal., 74(12):3884-3895, 2011.

[21] S. Hosseini and M. R. Pouryayevali. Nonsmooth optimization techniques on Riemannian manifolds. J. Optim. Theory Appl., 158(2):328-342, 2013.

[22] S. Hosseini and A. Uschmajew. A Riemannian Gradient Sampling Algorithm for Nonsmooth Optimization on Manifolds. SIAM J. Optim., 27(1):173-189, 2017.

[23] A. F. Izmailov and M. V. Solodov. Newton-type methods for optimization and variational problems. Springer Series in Operations Research and Financial Engineering. Springer, Cham, 2014.

[24] C. Lageman, U. Helmke, and J. H. Manton. The nonsmooth Newton method on Riemannian manifolds. Unpublished Notes (https://scholar.lib.vt.edu/MTNS/Papers/108.pdf).

[25] S. Lang. Differential and Riemannian manifolds, volume 160 of Graduate Texts in Mathematics. Springer-Verlag, New York, third edition, 1995.

[26] Y. S. Ledyaev and Q. J. Zhu. Nonsmooth analysis on smooth manifolds. Trans. Amer. Math. Soc., 359(8):3687-3732, 2007.

[27] J. M. Lee. Introduction to smooth manifolds, volume 218 of Graduate Texts in Mathematics. Springer, New York, second edition, 2013.

[28] C. Li and J. Wang. Newton's method on riemannian manifolds: Smale's point estimate theory under the $\gamma$-condition. IMA J. Numer. Anal., 26(2):228-251, 2006.

[29] R. Mifflin. Semismooth and semiconvex functions in constrained optimization. SIAM J. Control Optim., 15(6):959-972, 1977.

[30] J. M. Ortega and W. C. Rheinboldt. Iterative solution of nonlinear equations in several variables. Academic Press, New York-London, 1970.

[31] L. Qi and J. Sun. A nonsmooth version of Newton's method. Math. Programming, 58(3, Ser. A):353-367, 1993 .

[32] F. Rampazzo and H. J. Sussmann. Commutators of flow maps of nonsmooth vector fields. J. Differential Equations, 232(1):134-175, 2007. 
[33] T. Sakai. Riemannian geometry, volume 149 of Translations of Mathematical Monographs. American Mathematical Society, Providence, RI, 1996. Translated from the 1992 Japanese original by the author.

[34] M. Shub. Some remarks on dynamical systems and numerical analysis. In Dynamical systems and partial differential equations (Caracas, 1984), pages 69-91. Univ. Simon Bolivar, Caracas, 1986.

[35] S. T. Smith. Optimization techniques on Riemannian manifolds. In Hamiltonian and gradient flows, algorithms and control, volume 3 of Fields Inst. Commun., pages 113-136. Amer. Math. Soc., Providence, RI, 1994.

[36] M. Spivak. A comprehensive introduction to differential geometry. Vol. II. Publish or Perish, Inc., Wilmington, Del., third edition, 1999.

[37] L. W. Tu. An introduction to manifolds. Universitext. Springer-Verlag New York, second edition, 2011.

[38] C. Udriște. Convex functions and optimization methods on Riemannian manifolds, volume 297 of Mathematics and its Applications. Kluwer Academic Publishers Group, Dordrecht, 1994.

[39] J. H. Wang. Convergence of Newton's method for sections on Riemannian manifolds. J. Optim. Theory Appl., 148(1):125-145, 2011. 